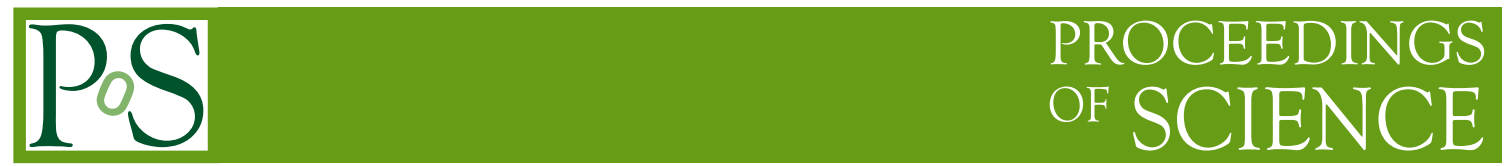

\title{
Heavy Ion Theory addressed in the above facilities
}

\author{
Néstor Armesto* \\ Departamento de Física de Partículas and IGFAE, \\ Universidade de Santiago de Compostela, 15782 Santiago de Compostela, Galicia-Spain \\ E-mail: nestor.armesto@usc.es
}

\begin{abstract}
In this contribution, I summarise some ideas regarding the impact of the study of electronproton/nucleus collisions on our understanding of heavy ion physics at high energies. It is based on a presentation during the panel discussion in DIS 2018 that was aimed to the elaboration of a document of the DIS community for the European Strategy for Particle Physics to be defined in 2020 .
\end{abstract}

XXVI International Workshop on Deep-Inelastic Scattering and Related Subjects (DIS2018)

16-20 April 2018

Kobe, Japan

${ }^{*}$ Speaker. 


\section{Introduction}

In view of the coming European Strategy for Particle Physics whose final result will appear in May 2020 [1], a panel discussion took place during DIS2018 with the final aim of elaborating a document of the DIS community to present for the Strategy discussion before the end of 2018. There, the electron-proton/nucleus facilities that are currently under discussion were presented $[2,3,4,5]$. Besides, the QCD aspects that could be addressed in such machines were analysed [6], together with those regarding heavy ion physics in my presentation. This is the corresponding writeup that will be very brief and contains uniquely my personal view on the subject. Due to space limitations, I will only point to some references containing either key ideas or reviews, but I will not intend to provide a full list of them. For more information, the reader should go to the web page of the Quark Matter 2018 Conference [7], held in May this year, where the latest discussions in the field took place.

\section{Present status of heavy ion physics}

Within our present understanding of heavy ion collisions at high energies as studied at RHIC [8] and the LHC [9, 10], the matter created there is very dense, with energies densities exceeding those predicted in lattice QCD for deconfinement and chiral symmetry restoration. Two main features of data, of relevance for the DIS community, are:

(i) Such matter shows collective features in the soft sector that are well described by relativistic hydrodynamics if applied very early (at times smaller than $1 \mathrm{fm} / \mathrm{c}$ ) after the collision, suggesting approximate equilibration or isotropisation. For its successful description of data, relativistic hydrodynamics must use a very small shear viscosity that points to strong interactions in the medium, opposed to the classical view of a plasma of quasi-free quarks and gluons. This fact has led to the paradigm of the perfect fluid created in heavy ion collisions.

(ii) It is extremely opaque to energetic partons or particles traversing it, as evident from the strong modification of the yield of hard probes (observables characterised by a large scale, thus computable in perturbative QCD) like high- $p_{T}$ particles, jets, quarkonia,...

\section{Open questions}

The most urgent open questions in the field are understanding [7]:

(a) Why the medium is describable by hydrodynamics (so early) [11] even in small systems, pp and pA (that show features that in nucleus-nucleus collisions are considered as characteristic of quark-gluon plasma formation, see e.g. [12]).

(b) How does it get approximately isotropised, and whether the relevant dynamics is weak or strong coupling [13].

(c) How to reduce the uncertainty in the extraction of QCD medium parameters, for which we need knowledge of the initial conditions for collective behaviour and of the modification of yields of perturbative processes in a coloured medium. 


\section{Future of the heavy ion programmes}

Waiting for electron-hadron/nucleus colliders, heavy ion collisions will run at RHIC [14] until mid-2020's, with detector improvements (particularly the new sPHENIX), a ten times increase in statistics and a Beam Energy Scan. There are also plans at the LHC, with a $\mathrm{PbPb}$ run in 2018, and heavy ions during Runs 3 and 4 (HL-LHC for ions, [15]), with the integrated luminosity goal of $10 \mathrm{nb}^{-1}$ per experiment ALICE, ATLAS and CMS. The current discussion at the LHC lies in how many pp and $\mathrm{pPb}$ runs besides $\mathrm{PbPb}$ ones would be desirable, and the possibility of colliding smaller ions and of a fixed target program [16] (in LHCb and ALICE, and other proposals like AFTER). While present $\mathrm{pPb}$ and UPC data from the LHC have not impacted largely the determination of PDFs, the possibilities with data in the forward rapidity region in $\mathrm{pPb}$ (with studies of new detectors like the FoCal at ALICE) and with UPCs are under discussion. In the longer term future, heavy ion programmes at the HE-LHC [17] and the FCC [5] are currently under discussion.

\section{What ep/eA can contribute}

ep and eA collisions will contribute to clarify many of the mentioned open points [2, 3, 4]:

- They will provide a detailed picture of the initial wave function of protons and nuclei, both the partonic densities and the transverse structure.

- They will clarify the mechanism of particle production, factorisation or not, both in DIS and in pp via a comparison with accurate parton densities.

- They will test the ideas about isotropisation that allows the use of hydrodynamics, by studying how small a system can become and still show collective features.

- They will characterise accurately the cold nuclear matter effects on the production cross sections of perturbative processes, hard probes, used for medium analysis in heavy ion collisions.

In order to achieve these goals, several requirements exist:

1) Concerning colliding species, both ep and eA are required. ep will establish the eventual new dynamics at small- $x$ [18] and set the reference for genuine nuclear effects. Then, the comparison of ep and eA will determine whether such new dynamics is a density effect like non-linear saturation, or a linear one like resummation.

2) Concerning kinematics and event rates:

- Large collision energy is required to explore the partonic structure of protons and nuclei and check the validity of the factorisation theorems in the relevant kinematic region for hh/AA colliders.

- Energy and luminosity are needed to be able to completely unfold the nuclear partonic structure using $\mathrm{NC}, \mathrm{CC}$ and heavy flavour decomposition, making it possible to extract the PDFs of a single nucleus without any resource to the proton PDFs - thus with nuclear effects defined a posteriori. 
- Energy will also make it possible to have a sizeable lever arm in $Q^{2}$ at small $x$ to study a new regime of QCD dominated by non-linear phenomena (named saturation) [18], and to explore rarer hard probes (tops, Higgs) that will play a role in heavy ion physics in future colliders like HE-LHC or FCC-hh [5].

Fig. 1 shows the regions of the kinematic plane presently explored in DIS, DY and neutrino nuclear experiments, and in $\mathrm{pPb}$ collisions at the $\mathrm{LHC}$, and to be explored in projected electron-ion colliders and in $\mathrm{pPb}$ collisions at the LHC and the FCC-hh.

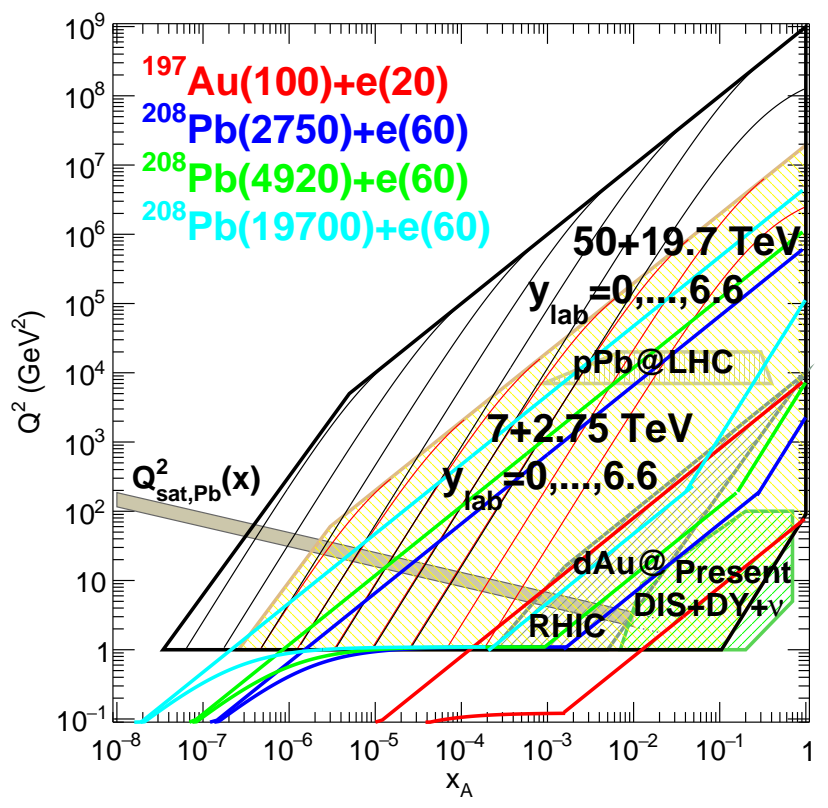

Figure 1: Regions of the $x-Q^{2}$ plane covered by present DIS, DY and neutrino nuclear experiments, by $\mathrm{pPb}$ collisions at the LHC, by projected electron-ion colliders and by $\mathrm{pPb}$ collisions at the LHC and the FCC-hh.

It becomes evident from the Figure that eA colliders will be complementary to the pA and UPC programmes at colliders, with larger kinematic reach but less precise due mainly to theoretical uncertainties intrinsic to those processes. They will also be complementary one each other. The EIC will partially overlap with the kinematic region previously measured in fixed target experiments, and has its focus on high precision measurements including varying nuclear species and particle identification down to low transverse momenta, being a versatile machine. The LHeC and FCC-eh, running concurrently with the respective hh or AA programs, will cover a much larger kinematic region, with access to very small $x$ and large $Q^{2}$ of relevance for future hh/AA colliders. This, together with the different additional rich physics programs of both machines, makes them crucial for the interpretation of the results in heavy ion collisions.

\section{Acknowledgments}

I thank the organisers for their invitation to provide this talk. I acknowledge financial support by Ministerio de Ciencia e Innovación of Spain under projects FPA2014-58293-C2-1-P, FPA2017- 
83814-P and Unidad de Excelencia María de Maetzu under project MDM-2016-0692, Xunta de Galicia (Consellería de Educación) within the Strategic Unit AGRUP2015/11, and FEDER. This work has been performed in the framework of COST Action CA15213 "Theory of hot matter and relativistic heavy ion collisions" (THOR).

\section{References}

[1] A. Levy, panel talk at this conference, https://indico.cern.ch/event/656250/contributions/2939085/.

[2] B. Surrow, panel talk at this conference, https://indico.cern.ch/event/656250/contributions/2939330/.

[3] M. Klein, panel talk at this conference, https://indico.cern.ch/event/656250/contributions/2939331/.

[4] A. C. Caldwell, panel talk at this conference, https://indico.cern.ch/event/656250/contributions/2939332/.

[5] D. d'Enterría, panel talk at this conference, https://indico.cern.ch/event/656250/contributions/2939333/.

[6] J. W. Qiu, panel talk at this conference, https://indico.cern.ch/event/656250/contributions/2939334/.

[7] Quark Matter 2018, Venice, May 13th-19th 2018, https://indico.cern.ch/event/656452/.

[8] M. Gyulassy and L. McLerran, "New forms of QCD matter discovered at RHIC," Nucl. Phys. A $\mathbf{7 5 0}$ (2005) 30 [nucl-th/0405013].

[9] N. Armesto and E. Scomparin, "heavy ion collisions at the Large Hadron Collider: a review of the results from Run 1,” Eur. Phys. J. Plus 131 (2016) no.3, 52 [arXiv:1511.02151 [nucl-ex]].

[10] C. A. Salgado and J. P. Wessels, "Proton?Lead Collisions at the CERN LHC," Ann. Rev. Nucl. Part. Sci. 66 (2016) 449.

[11] R. D. Weller and P. Romatschke, "One fluid to rule them all: viscous hydrodynamic description of event-by-event central $\mathrm{p}+\mathrm{p}, \mathrm{p}+\mathrm{Pb}$ and $\mathrm{Pb}+\mathrm{Pb}$ collisions at $\sqrt{s}=5.02 \mathrm{TeV}$," Phys. Lett. B 774 (2017) 351 [arXiv:1701.07145 [nucl-th]].

[12] C. Loizides, "Experimental overview on small collision systems at the LHC," Nucl. Phys. A 956 (2016) 200 [arXiv:1602.09138 [nucl-ex]].

[13] P. Romatschke, "Do nuclear collisions create a locally equilibrated quark?gluon plasma?," Eur. Phys. J. C 77 (2017) no.1, 21 [arXiv:1609.02820 [nucl-th]].

[14] B. Müller, "The Future of RHIC," PoS KMI 2017 (2017) 013.

[15] J. Jowett at the LHC Performance Workshop 2017, Chamonix, January 23rd-27th 2017, https://indico.cern.ch/event/580313/contributions/2359507/.

[16] C. Hadjidakis et al., "A Fixed-Target Programme at the LHC: Physics Case and Projected Performances for Heavy-Ion, Hadron, Spin and Astroparticle Studies," arXiv:1807.00603 [hep-ex].

[17] Workshop on the physics of HL-LHC, and perspectives at HE-LHC, CERN, June 18th-20th 2018, https://indico.cern.ch/event/686494/timetable/.

[18] Y. V. Kovchegov and E. Levin, "Quantum chromodynamics at high energy," Camb. Monogr. Part. Phys. Nucl. Phys. Cosmol. 33 (2012). 